Pacific Journal of Mathematic 


\title{
A GEOMETRIC INEQUALITY WITH APPLICATIONS TO LINEAR FORMS
}

\author{
JEFFREY D. VAALER
}

Let $C_{N}$ be a cube of volume one centered at the origin in $\boldsymbol{R}^{N}$ and let $P_{K}$ be a $K$-dimensional subspace of $\boldsymbol{R}^{N}$. We prove that $C_{N} \cap P_{K}$ has $K$-dimensional volume greater than or equal to one. As an application of this inequality we obtain a precise version of Minkowski's linear forms theorem. We also state a conjecture which would allow our method to be generalized.

1. Introduction. Let $C_{N}=[-1 / 2,1 / 2]^{N}$ be the $N$-dimensional cube of volume one centered at the origin in $\boldsymbol{R}^{N}$ and suppose that $P_{K}$ is a $K$-dimensional linear subspace of $\boldsymbol{R}^{N}$. Dr. Anton Good has conjectured that the $K$-dimensional volume of $P_{K} \cap C_{N}$ is always greater than or equal to one. In case $K=N-1$ this has recently been proved by Hensley [6], who also obtained upper bounds for this volume. Our purpose in this paper is to prove the conjecture for arbitrary $K$ and to give some applications to Minkowski's theorem on linear forms. In fact we prove a more general inequality for the product of spheres of various dimensions which contains the conjecture as a special case.

We write $\bar{x}$ for the column vector $\left(\begin{array}{c}x_{1} \\ \cdots \\ x_{n}\end{array}\right)$ in $\boldsymbol{R}^{n}$ and

$$
|\bar{x}|=\left(\sum_{j=1}^{n}\left(x_{j}\right)^{2}\right)^{1 / 2}
$$

for its length. We define the sphere $S_{n}$ by

$$
S_{n}=\left\{\bar{x} \in \boldsymbol{R}^{n}:|\bar{x}| \leqq \rho_{n}\right\}
$$

where $\rho_{n}=\pi^{-1 / 2}\{\Gamma(n / 2+1)\}^{1 / n}$. It follows that $\mu_{n}\left(S_{n}\right)=1$ where $\mu_{n}$ is Lebesgue measure on $\boldsymbol{R}^{n}$. Also we let $\chi_{U}(\bar{x})$ denote the characteristic function of a subset $U$ in $\boldsymbol{R}^{n}$.

Our first main result is contained in the following theorem.

THEOREM 1. Suppose that $n_{1}, n_{2}, \cdots, n_{J}$ are positive integers, $Q_{N}=S_{n_{1}} \times S_{n_{2}} \times \cdots \times S_{n_{J}}$ is in $\boldsymbol{R}^{N}, N=n_{1}+n_{2}+\cdots+n_{J}$, and $A$ is a real $N \times K$ matrix, $\operatorname{rank}(A)=K$. Then

$$
\left|\operatorname{det} A^{T} A\right|^{-1 / 2} \leqq \int_{R^{K}} \chi_{Q_{N}}(A \bar{x}) d \mu_{K}(\bar{x}),
$$

where $A^{T}$ is the transpose of $A$. 
We note that if $\operatorname{rank}(A)<K$ then each side of (1.1) is infinite. From Theorem 1 we easily deduce a lower bound for $\mu_{K}\left(Q_{N} \cap P_{K}\right)$.

Corollary. Let $Q_{N}$ be as in Theorem 1 and let $P_{K}$ be a $K$ dimensional subspace of $\boldsymbol{R}^{N}$. Then $\mu_{K}\left(Q_{N} \cap P_{K}\right) \geqq 1$.

Proof. Choose $A$ in Theorem 1 so that the columns of $A$ form an orthonormal basis for $P_{K}$ in $\boldsymbol{R}^{N}$. Then the left hand side of (1.1) is 1 while the right hand side is $\mu_{K}\left(Q_{N} \cap P_{K}\right)$.

The corollary clearly contains Good's conjecture since $Q_{N}=C_{N}$ if $n_{j}=1$ and $J=N$.

Next we suppose that $L_{j}(\bar{x}), j=1,2, \cdots, N$ are $N$ linear forms in $K$ variables,

$$
L_{j}(\bar{x})=\sum_{k=1}^{K} a_{j k} x_{k},
$$

so that $A=\left(a_{j k}\right)$ is an $N \times K$ matrix. We assume that the forms $L_{j}$ are real for $j=1,2, \cdots, r$ and that the remaining forms consist of $s$ pairs of complex conjugate forms arranged so that $L_{r+2 j-1}=\bar{L}_{r+2 j}$ for $j=1,2, \cdots, s$. Thus $N=r+2 s$. Let $\varepsilon_{1}, \varepsilon_{2}, \cdots, \varepsilon_{N}$ be positive with $\varepsilon_{r+2 j-1}=\varepsilon_{r+2 j}$ for $j=1,2, \cdots, s$. We define the $N \times N$ diagonal matrix $E$ by $E=\left(c_{j} \delta_{j k}\right)$ where $c_{j}=\varepsilon_{j}^{-1}$ if $j=1,2, \cdots, r, c_{j}=(2 / \pi)^{1 / 2} \varepsilon_{j}^{-1}$ if $j=r+1, r+2, \cdots, N$ and $\delta_{j k}$ is the Kronecker delta. Theorem 1 allows us to prove the following precise version of Minkowski's classical result on linear forms.

THEOREM 2. Let $M$ be a positive integer and suppose that

$$
M\left|\operatorname{det} A^{*} E^{2} A\right|^{1 / 2} \leqq 1,
$$

where $A^{*}$ is the complex conjugate transpose of the matrix $A$. Then there exist at least $M$ distinct pairs of nonzero lattice points $\pm \bar{v}_{m}$, $m=1,2, \cdots, M$, such that

$$
\left|L_{j}\left( \pm \bar{v}_{m}\right)\right| \leqq \varepsilon_{j}
$$

for each $j$ and each $m$. In particular if $\left|\operatorname{det} A^{*} A\right|>0$ then there exists a pair of nonzero lattice points $\pm \bar{v}$ such that

$$
\left|L_{j}( \pm \bar{v})\right| \leqq\left|\operatorname{det} A^{*} A\right|^{1 / 2 K}
$$

for $j=1,2, \cdots, r$, and

$$
\left.\left|L_{j}( \pm \bar{v}) \leqq\left(\frac{2}{\pi}\right)^{1 / 2}\right| \operatorname{det} A^{*} A\right|^{1 / 2 K}
$$

for $j=r+1, r+2, \cdots, N$. 
Theorem 2 was first proved in the case $N \leqq K$ and $M=1$ by Minkowski [8, p. 104]. Subsequently the extension of Minkowski's convex body theorem by van der Corput [5] allowed Theorem 2 to be proved for $N \leqq K$ and arbitrary $M$. Of course if $N=K$ then (1.2) becomes the more familiar condition

$$
M\left(\frac{2}{\pi}\right)^{s}|\operatorname{det} A| \leqq \varepsilon_{1} \varepsilon_{2} \cdots \varepsilon_{N},
$$

and if $N<K$ then (1.2) is trivially satisfied since the left hand side is zero. The novelty in our result is that Theorem 2 now holds for $1 \leqq K<N$. Previously in the case $1 \leqq K<N$ we knew only that (1.3) held if

$$
2^{K} M \leqq \mu_{K}\left(\left\{\bar{x} \in \boldsymbol{R}^{K}:\left|L_{j}(\bar{x})\right| \leqq \varepsilon_{j}, j=1,2, \cdots, N\right\}\right) .
$$

We prove Theorem 2 by showing that the right hand side of (1.6) is bounded from below by $2^{K}\left|\operatorname{det} A^{*} E^{2} A\right|^{-1 / 2}$. As will be clear from the proof, Theorem 2 could be generalized to include linear forms with values in $\boldsymbol{R}^{n}$ for various $n$.

In $\S 5$ we state a conjecture which would allow us to obtain a significant improvement in Theorem 1 . Specifically, we deduce from this conjecture an analogue of Theorem 1 in which $Q_{N}$ is replaced by an arbitrary closed, convex, symmetric subset of $\boldsymbol{R}^{N}$ having $N$ dimensional volume equal to one.

The author wishes to thank Professors Patrick Brockett, Douglas Hensley, and Bruce Palka for several helpful discussions on the subject of this paper.

2. Preliminary results. In this section we briefly summerize some facts about logarithmically concave measures and functions. A more detailed discription can be found in the papers of Kanter [7] and Prékopa [9].

A function $f: \boldsymbol{R}^{n} \rightarrow[0, \infty)$ is said to be log-concave if for every pair of vectors $\bar{x}_{1}, \bar{x}_{2}$ in $\boldsymbol{R}^{n}$ and every $\lambda, 0<\lambda<1$, we have

$$
f\left(\lambda \bar{x}_{1}+(1-\lambda) \bar{x}_{2}\right) \geqq\left(f\left(\bar{x}_{1}\right)\right)^{\lambda}\left(f\left(\bar{x}_{2}\right)\right)^{1-\lambda} .
$$

A probability measure $\nu$ defined on the measurable subsets of $\boldsymbol{R}^{n}$ is log-concave if for every pair of open convex sets $U_{1}$ and $U_{2}$ in $\boldsymbol{R}^{n}$ and every $\lambda, 0<\lambda<1$, we have

$$
\nu\left(\lambda U_{1}+(1-\lambda) U_{2}\right) \geqq\left(\nu\left(U_{1}\right)\right)^{\lambda}\left(\nu\left(U_{2}\right)\right)^{1-\lambda},
$$

where + on the left hand side of (2.1) indicates Minkowski addition of sets. Clearly (2.1) holds for all open convex sets $U_{1}$ and $U_{2}$ if and only if it holds for all closed convex sets $U_{1}$ and $U_{2}$. The relationship 
between log-concave measures and log-concave functions is contained in the following lemma.

LeMma 3. Let $\nu$ be a log-concave probability measure on $\boldsymbol{R}^{n}$ and suppose that the support of $\nu$ spans the $k$-dimensional subspace $P_{k}$ in $\boldsymbol{R}^{n}$. Then there is a log-concave probability density function $f$ defined on $P_{k}$ such that $d \nu=f d \mu_{k}$, where $\mu_{k}$ is $k$-dimensional Lebesgue measure on $P_{k}$. Conversely for any log-concave probability density function $f$ defined on a $k$-dimensional subspace $P_{k}$ in $\boldsymbol{R}^{n}$, the probability measure defined by $d \nu=f d \mu_{k}$ is log-concave, where $\mu_{k}$ is Lebesgue measure on $P_{k}$.

The first part of Lemma 3 is a result of Borell [2, p. 123] while the converse was proved by Prékopa [9], (see also Kanter [7, Lemma 2.1]).

Let $\nu_{1}$ and $\nu_{2}$ be probability measures on $\boldsymbol{R}^{n}$. We say that $\nu_{2}$ is more peaked than $\nu_{1}$ if

$$
\nu_{1}(U) \leqq \nu_{2}(U)
$$

for all closed, convex, symmetric subsets $U$ in $\boldsymbol{R}^{n}$. (We recall that $U \subseteq \boldsymbol{R}^{n}$ is symmetric if $U=-U$.) If $f_{1}$ and $f_{2}$ are probability density functions on $\boldsymbol{R}^{n}$ we say that $f_{2}$ is more peaked than $f_{1}$ if the measure $f_{2} d \mu_{n}$ is more peaked than the measure $f_{1} d \mu_{n}$. The notion of peakedness was introduced by Birnbaum [1] and Sherman [10]. A complementary relation is that of symmetric dominance in the sense of Kanter [7]. If $\nu_{3}$ and $\nu_{4}$ are measures on $\boldsymbol{R}^{n}$ then $\nu_{3}$ symmetrically dominates $\nu_{4}$ if

$$
\nu_{3}\left(\boldsymbol{R}^{n} \backslash U\right) \geqq \nu_{4}\left(\boldsymbol{R}^{n} \backslash U\right)
$$

for all closed, convex, symmetric subsets $U$ in $\boldsymbol{R}^{n}$. It is clear that if $\nu_{3}$ and $\nu_{4}$ are both probability measures then $\nu_{3}$ symmetrically dominates $\nu_{4}$ if and only if $\nu_{4}$ is more peaked than $\nu_{3}$. For our purposes it is more convenient to work with the relation of peakedness.

If $\nu_{1}$ and $\nu_{2}$ are log-concave probability measures on $\boldsymbol{R}^{n}$ then the convolution $\nu_{1}^{*} \nu_{2}$ is also log-concave on $\boldsymbol{R}^{n}$ (Kanter [7, Lemma 2.3]). It follows that if $\nu_{1}$ and $\nu_{2}$ are log-concave probability measures on $\boldsymbol{R}^{n_{1}}$ and $\boldsymbol{R}^{n_{2}}$ respectively then the product measure $\nu_{1} \times \nu_{2}$ is logconcave on $\boldsymbol{R}^{n_{1}} \times \boldsymbol{R}^{n_{2}}$. Forming product measures also preserves the peakedness relation.

Lemma 4. Suppose that $\nu_{1}, \nu_{2}, \nu_{1}^{\prime}$ and $\nu_{2}^{\prime}$ are all log-concave probability measures such that $\nu_{1}$ is mome peaked than $\nu_{1}^{\prime}$ on $\boldsymbol{R}^{n_{1}}$ and 
$\nu_{2}$ is more peaked than $\nu_{2}^{\prime}$ on $\boldsymbol{R}^{n_{2}}$. Then $\nu_{1} \times \nu_{2}$ is more peaked than $\nu_{1}^{\prime} \times \nu_{2}^{\prime}$ on $\boldsymbol{R}^{n_{1}} \times \boldsymbol{R}^{n_{2}}$.

For the proof of Lemma 4 we refer to Kanter [7, Corollary 3.2] where the result is obtained for the more general class of unimodal measures.

3. Proof of Theorem 1. We begin by proving the following lemma.

Lemma 5. Suppose that $n_{1}, n_{2}, \cdots, n_{J}$ are positive integers and $Q_{N}=S_{n_{1}} \times S_{n_{2}} \times \cdots \times S_{n_{J}}$ is in $\boldsymbol{R}^{N}, N=n_{1}+n_{2}+\cdots+n_{J}$. Then $\chi_{Q_{N}}(\bar{x})$ is more peaked than the normal density function $\exp \left\{-\pi|\bar{x}|^{2}\right\}$ on $\boldsymbol{R}^{x}$.

Proof. Since the measures $\chi_{Q_{N}}(\bar{x}) d \mu_{N}(\bar{x})$ and $\exp \left\{-\pi|\bar{x}|^{2}\right\} d \mu_{N}(\bar{x})$ are both product measures which factor in $\boldsymbol{R}^{n_{1}} \times \boldsymbol{R}^{n_{2}} \times \cdots \times \boldsymbol{R}^{n_{J}}$ it suffices to prove the peakedness relation in each factor space and then apply Lemma 4. Thus we need only show that for each positive integer $n, \chi_{S_{n}}(\bar{x})$ is more peaked than $\exp \left\{-\pi|\bar{x}|^{2}\right\}$ on $\boldsymbol{R}^{n}$. Of course it is trivial to verify that both of the density functions $\chi_{S_{n}}(\bar{x})$ and $\exp \left\{-\pi|\bar{x}|^{2}\right\}$ are log-concave on $\boldsymbol{R}^{n}$.

Let $\sum_{n-1}=\left\{\bar{x} \in \boldsymbol{R}^{n}:|\bar{x}|=1\right\}$ so that for each $\bar{x} \neq \overline{0}$ in $\boldsymbol{R}^{n}$ we have the unique polar decomposition $\bar{x}=r \bar{x}^{\prime}$ where $r=|\bar{x}|$ and $\bar{x}^{\prime} \in \sum_{n-1}$. If $U$ is a closed, convex, symmetric subset of $\boldsymbol{R}^{n}$ then it follows that

$$
\int_{U} \exp \left\{-\pi|\bar{x}|^{2}\right\} d \mu_{n}(\bar{x})=\int_{\Sigma_{n-1}} \int_{0}^{\infty} \chi_{U}\left(r \bar{x}^{\prime}\right) \exp \left\{-\pi r^{2}\right\} r^{n-1} d r d \bar{x}^{\prime},
$$

where $d \bar{x}^{\prime}$ is the induced Lebesgue measure on $\sum_{n-1}$. Now for each fixed $\bar{x}^{\prime} \in \sum_{n-1}$ we have either

$$
\chi_{L^{\prime}}\left(r \bar{x}^{\prime}\right) \leqq \chi_{S_{n}}\left(r \bar{x}^{\prime}\right), \quad 0 \leqq r<\infty
$$

or

$$
\chi_{S_{n}}\left(r \bar{x}^{\prime}\right) \leqq \chi_{U}\left(r \bar{x}^{\prime}\right), \quad 0 \leqq r<\infty,
$$

since $S_{n}$ and $U$ are convex. If (3.2) holds at $\bar{x}^{\prime}$ then

$$
\begin{aligned}
& \int_{0}^{\infty} \chi_{U}\left(r \bar{x}^{\prime}\right) \exp \left\{-\pi r^{2}\right\} r^{n-1} d r \\
& \quad \leqq \int_{0}^{\infty} \chi_{V}\left(r \bar{x}^{\prime}\right) r^{n-1} d r=\int_{0}^{\infty} \chi_{U}\left(r \bar{x}^{\prime}\right) \chi_{S_{n}}\left(r \bar{x}^{\prime}\right) r^{n-1} d r
\end{aligned}
$$

If (3.3) holds at $\bar{x}^{\prime}$ then 


$$
\begin{aligned}
& \int_{0}^{\infty} \chi_{U}\left(r \bar{x}^{\prime}\right) \exp \left\{-\pi r^{2}\right\} r^{n-1} d r \\
& \quad \leqq \int_{0}^{\infty} \exp \left\{-\pi r^{2}\right\} r^{n-1} d r=n^{-1} \pi^{-n / 2} \Gamma\left(\frac{n}{2}+1\right) \\
& \quad=\int_{0}^{\infty} \chi_{S_{n}}\left(r \bar{x}^{\prime}\right) r^{n-1} d r \\
& \quad=\int_{0}^{\infty} \chi_{U}\left(r \bar{x}^{\prime}\right) \chi_{S_{n}}\left(r \bar{x}^{\prime}\right) r^{n-1} d r .
\end{aligned}
$$

Combining (3.1), (3.4) and (3.5) we obtain

$$
\int_{U} \exp \left\{-\pi|\bar{x}|^{2}\right\} d \mu_{n}(\bar{x}) \leqq \int_{\Sigma_{n-1}} \int_{0}^{\infty} \chi_{U}\left(r \bar{x}^{\prime}\right) \chi_{S_{n}}\left(r \bar{x}^{\prime}\right) r^{n-1} d r d \bar{x}^{\prime}=\int_{U} \chi_{S_{n}}(\bar{x}) d \mu_{n}(\bar{x}) .
$$

Thus $\chi_{S_{n}}(\bar{x})$ is more peaked than $\exp \left\{-\pi|\bar{x}|^{2}\right\}$ on $R^{n}$ and the lemma is proved.

We now prove Theorem 1. If $N=K$ then (1.1) is trivial so we may suppose that $K^{\prime}=N-K$ is positive. Let $P_{K}$ be the $K$-dimensional subspace of $R^{N}$ spanned by the columns of $A$. Next let $W$ be an $N \times N$ matrix whose first $K$ columns are the columns of $A$ and whose next $K^{\prime}$ columns are the columns of an $N \times K^{\prime}$ matrix $B$. We choose the columns of $B$ so that they form an orthonormal basis in $\boldsymbol{R}^{N}$ of the $K^{\prime}$-dimensional subspace which is orthogonal to $P_{K}$. Identifying $\boldsymbol{R}^{N}$ with $\boldsymbol{R}^{K} \times \boldsymbol{R}^{K^{\prime}}$ we may write each $\bar{z} \in \boldsymbol{R}^{N}$ as $\bar{z}=(\bar{x} / \bar{y})$ where $\bar{x} \in \boldsymbol{R}^{K}$ and $\bar{y} \in \boldsymbol{R}^{K^{\prime}}$. For each $\varepsilon, 0<\varepsilon \leqq 1$ we define

$$
H_{\varepsilon}=\left\{\bar{z} \in \boldsymbol{R}^{N}: z=\left(\frac{\bar{x}}{\bar{y}}\right), \max _{1 \leqq j \leqq K^{\prime}}\left|y_{j}\right| \leqq \frac{\varepsilon}{2}\right\}
$$

and

$$
H_{\varepsilon}^{\prime}=\left\{\bar{y} \in \boldsymbol{R}^{K^{\prime}}: \max _{1 \leqq j \leqq K^{\prime}}\left|y_{j}\right| \leqq \frac{\varepsilon}{2}\right\} .
$$

Clearly $H_{\varepsilon}$ is a closed, convex, symmetric subset of $\boldsymbol{R}^{N}$ and so is the image of $H_{\varepsilon}$ under the nonsingular linear transformation determined by $W$. Thus by Lemma 5 ,

$$
\int_{H_{\varepsilon}} \exp \left\{-\pi|W \bar{z}|^{2}\right\} d \mu_{N}(\bar{z}) \leqq \int_{H_{\varepsilon}} \chi_{Q_{N}}(W \bar{z}) d \mu_{N}(\bar{z}) .
$$

Multiplying each side of (3.6) by $\left\{\mu_{K^{\prime}}\left(H_{\varepsilon^{\prime}}^{\prime}\right)\right\}^{-1}=\varepsilon^{-K^{\prime}}$ and factoring $H_{8}$ into $\boldsymbol{R}^{K} \times H_{\varepsilon}^{\prime}$ we find that

$$
\begin{aligned}
& \varepsilon^{-K^{\prime}} \int_{\boldsymbol{R}^{K}} \int_{H_{\varepsilon}^{\prime}} \exp \left\{-\pi|A \bar{x}+B \bar{y}|^{2}\right\} d \mu_{K^{\prime}}(\bar{y}) d \mu_{K}(\bar{x}) \\
& \quad \leqq \varepsilon^{-K^{\prime}} \int_{\boldsymbol{R}^{K}} \int_{H_{\varepsilon}^{\prime}} \chi_{Q_{N}}(A \bar{x}+B \bar{y}) d \mu_{K^{\prime}}(\bar{y}) d \mu_{K}(\bar{x}) .
\end{aligned}
$$


By the orthogonality condition $|A \bar{x}+B \bar{y}|^{2}=|A \bar{x}|^{2}+|B \bar{y}|^{2}$ and so as $\varepsilon \rightarrow 0+$ the left hand side of (3.7) clearly converges to

$$
\int_{R K} \exp \left\{-\pi|A \bar{x}|^{2}\right\} d \mu_{K}(\bar{x})=\left|\operatorname{det} A^{T} A\right|^{-1 / 2} .
$$

To evaluate the corresponding limit on the right hand side of (3.7) we observe that for $0<\varepsilon \leqq 1$ and each $\bar{x} \in \boldsymbol{R}^{K}$,

$$
\varepsilon^{-K^{\prime}} \int_{H_{\varepsilon}^{\prime}} \chi_{Q_{N}}(A \bar{x}+B \bar{y}) d \mu_{K^{\prime}}(\bar{y}) \leqq 1 .
$$

Since $Q_{. V}$ and $H_{\varepsilon}^{\prime}$ are both bounded we have

$$
\varepsilon^{-K^{\prime}} \int_{H_{\varepsilon}^{\prime}} \chi_{Q_{N}}(A \bar{x}+B \bar{y}) d \mu_{K^{\prime}}(\bar{y})=0
$$

for sufficiently large $|\bar{x}|$ independent of $\varepsilon$. Thus by dominated convergence the limit on the right of (3.7) as $\varepsilon \rightarrow 0+$ is

$$
\int_{R^{K}}\left\{\lim _{\varepsilon \rightarrow 0+} \varepsilon^{-K^{\prime}} \int_{H_{\varepsilon}^{\prime}} \chi_{Q_{N}}(A \bar{x}+B \bar{y}) d \mu_{K^{\prime}}(\bar{y})\right\} d \mu_{K}(\bar{x}) \text {. }
$$

Clearly

$$
\lim _{\varepsilon \rightarrow 0+} \varepsilon^{-K^{\prime}} \int_{H_{\varepsilon}^{\prime}} \chi_{Q_{N}}(A \bar{x}+B \bar{y}) d \mu_{K^{\prime}}(\bar{y})=\chi_{Q_{N}}(A \bar{x})
$$

except possibly when $A \bar{x}$ is a boundary point of $Q_{N} \cap P_{K}$, Since this boundary has $K$-dimensional measure zero we see that (3.8) is equal to

$$
\int_{R^{K}} \chi_{Q_{N}}(A \bar{x}) d \mu_{K}(\bar{x})
$$

We have now shown that as $\varepsilon \rightarrow 0+$ on each side of (3.7) we obtain (1.1) and this proves the theorem.

4. Proof of Theorem 2. By van der Corput's extension of Minkowski's convex body theorem [5] (see also Cassels [4, Chapter III, Theorem II]) the condition (1.6) implies that there exist at least $M$ distinct pairs $\pm \bar{v}_{m}, m=1,2, \cdots, M$, of nonzero lattice points such that (1.3) holds. If $\operatorname{rank}(A)<K$ then (1.2) and (1.6) are both trivially satisfied. Thus to eatablish the first part of Theorem 2 it suffices to show that if $\operatorname{rank}(A)=K$ then

(4.1) $2^{K}\left|\operatorname{det} A^{*} E^{2} A\right|^{-1 / 2} \leqq \mu_{K}\left(\left\{\bar{x} \in R^{K}:\left|L_{j}(\bar{x})\right| \leqq \varepsilon_{j}, j=1,2, \cdots, N\right\}\right)$.

Let $G_{j}(\bar{x}), j=1,2, \cdots, N$ be linear forms defined by $G_{j}(\bar{x})=L_{j}(\bar{x})$ for $j=1,2, \cdots, r$ and 


$$
\begin{aligned}
G_{r+2 j-1}(\bar{x}) & =\sqrt{2} \operatorname{Re}\left\{L_{r+2 j-1}(\bar{x})\right\}, \\
G_{r+2 j}(\bar{x}) & =\sqrt{2} \operatorname{Im}\left\{L_{r+2 j-1}(\bar{x})\right\}
\end{aligned}
$$

for $j=1,2, \cdots, s$. We write $B=\left(b_{j k}\right)$ for the corresponding real $N \times K$ matrix so that

$$
G_{j}(\bar{x})=\sum_{k=1}^{K} b_{j k} x_{k} .
$$

Next we let $Q_{N}=S_{n_{1}} \times S_{n_{2}} \times \cdots \times S_{n_{r+s}}$ where $n_{j}=1$ for $j=$ $1,2, \cdots, r$ and $n_{j}=2$ for $j=r+1, r+2, \cdots, r+s$. It follows that $\left|L_{j}(\bar{x})\right| \leqq \varepsilon_{j}$ if and only if $1 / 2 \varepsilon_{j}^{-1} G_{j}(\bar{x}) \in S_{n_{j}}, j=1,2, \cdots, r$, and

$$
\left|L_{r+2 j-1}(\bar{x})\right|=\left|L_{r+2 j}(\bar{x})\right| \leqq \varepsilon_{r+2 j}
$$

if and only if

$$
(2 \pi)^{-1 / 2} \varepsilon_{r+2 j}^{-1}\left(\begin{array}{l}
G_{r+2 j-1}(\bar{x}) \\
G_{r+2 j}(\bar{x})
\end{array}\right) \in S_{n_{r+j}},
$$

$j=1,2, \cdots, s . \quad$ Therefore

$$
\begin{aligned}
& \mu_{K}(\{\bar{x}\left.\left.\in \boldsymbol{R}^{K}:\left|L_{j}(\bar{x})\right| \leqq \varepsilon_{j}, j=1,2, \cdots, N\right\}\right) \\
&=\mu_{K}\left(\left\{\bar{x} \in R^{K}: \frac{1}{2} E B \bar{x} \in Q_{N}\right\}\right)=\int_{R^{K}} \chi_{Q_{N}}\left(\frac{1}{2} E B \bar{x}\right) d \mu_{K}(\bar{x}) \\
& \geqq\left|\operatorname{det}\left(\frac{1}{2} E B\right)^{T}\left(\frac{1}{2} E B\right)\right|^{-1 / 2}=2^{K}\left|\operatorname{det} B^{T} E^{2} B\right|^{-1 / 2} .
\end{aligned}
$$

An easy computation shows that $B^{T} E^{2} B=A^{*} E^{2} A$ and so completes the proof of (4.1).

To prove the second part of Theorem 2 we choose $\varepsilon_{j}=\left|\operatorname{det} A^{*} A\right|^{1 / 2 K}$ for $j=1,2, \cdots, r$ and $\varepsilon_{j}=(2 / \pi)^{1 / 2}\left|\operatorname{det} A^{*} A\right|^{1 / 2 K}$ for $j=r+1, r+2$, $\cdots, N$. Then

$$
\left|\operatorname{det} A^{*} E^{2} A\right|=1
$$

and so (1.4) and (1.5) follow from the first part of the theorem.

5. Lower bounds for arbitrary convex bodies. In this section we suppose that $Q_{N}$ is a closed, convex, symmetric subset of $\boldsymbol{R}^{N}$ with $\mu_{N}\left(Q_{N}\right)=1$. If $A$ is an $N \times K$ matrix, $\operatorname{rank}(A)=K$, we will be interested in the problem of finding a lower bound for

$$
\int_{R^{K}} \chi_{Q_{N}}(A \bar{x}) d \mu_{K}(\bar{x})
$$

The method used to deduce Theorem 1 from Lemma 5 will also lead to a lower bound in this more general situation, provided that we 
can find a suitable normal density function on $\boldsymbol{R}^{N}$ which is less peaked than $\chi_{Q_{N}}(\bar{x})$. We succeeded in proving Lemma 5 because the special structure imposed on $Q_{N}$ allowed us to appeal to Lemma 4 . We now describe an alternative method which leads to a conjectured lower bound for (5.1).

We write $Q$ for $Q_{N}$ and we assume that $Q$ is a fixed, closed, convex, symmetric subset of $\boldsymbol{R}^{N}, \mu_{N}(Q)=1$. For each positive integer $m$ let

$$
\chi_{Q}^{(m)}(\bar{x})=\chi_{Q}^{*} \chi_{Q}^{*} \ldots \chi_{Q}(\bar{x})
$$

be the $m$-fold convolution of $\chi_{Q}$. We define the dilation operator $D_{\lambda}$ for $\lambda>0$ and for integrable real valued functions $f$ on $\boldsymbol{R}^{N}$ by

$$
D_{\lambda}(f)(\bar{x})=\lambda^{N} f(\lambda \bar{x}) .
$$

Next we define a sequence of positive numbers $\lambda_{m}, m=1,2, \cdots$ by

$$
\left(\lambda_{m}\right)^{N} \chi_{Q}^{(m)}(\overline{0})=1 \text {. }
$$

With this notation we have the following

ConJeCtURE 6. For each positive integer $m, \chi_{Q}(\bar{x})$ is more peaked than $D_{\lambda_{m}}\left(\chi_{Q}^{(m)}\right)(\bar{x})$.

Now let $\Omega$ be the $N \times N$ covariance matrix determined by a random vector which is uniformly distributed on the convex body $Q$. That is $\Omega=\left(\omega_{r s}\right)$ is the $N \times N$ matrix defined by

$$
\omega_{r s}=\int_{R^{N}} y_{r} y_{s} \chi_{Q}(\bar{y}) d \mu_{N}(\bar{y}),
$$

where $y_{r}$ and $y_{s}$ are the $r$ th and sth co-ordinate functions of $\bar{y}, r=$ $1,2, \cdots, N$, and $s=1,2, \cdots, N$. It is clear that $\Omega$ is symmetric and nonsingular since $Q$ has a nonempty interior. By the Central Limit Theorem (Breiman [3, Theorem 11.10]) we have

$$
\lim _{m \rightarrow \infty} D_{\sqrt{m}}\left(\chi_{Q}^{(m)}\right)(\bar{x})=(2 \pi)^{-N / 2}(\operatorname{det} \Omega)^{-1 / 2} \exp \left\{-\frac{1}{2} \bar{x}^{T} \Omega^{-1} \bar{x}\right\}
$$

uniformly for $x \in \boldsymbol{R}^{N}$. It follows that

$$
\lim _{m \rightarrow \infty} \frac{\lambda_{m}}{\sqrt{m}}=(2 \pi)^{1 / 2}(\operatorname{det} \Omega)^{1 / 2 N}
$$

and hence

$$
\lim _{m \rightarrow \infty} D_{\lambda_{m}}\left(\chi_{Q}^{(m)}\right)(\bar{x})=\exp \left\{-\pi(\operatorname{det} \Omega)^{1 / N} \bar{x}^{T} \Omega^{-1} \bar{x}\right\}
$$

uniformly for $x \in \boldsymbol{R}^{N}$. If the Conjecture 6 is true then for each 
positive integer $m$ and each closed, convex, symmetric subset $U$ of $\boldsymbol{R}^{N}$

$$
\int_{U} D_{\lambda_{m}}\left(\chi_{Q}^{(m)}\right)(\bar{x}) d \mu_{N}(\bar{x}) \leqq \int_{U} \chi_{Q}(\bar{x}) d \mu_{N}(\bar{x}) .
$$

Letting $m \rightarrow \infty$ on the left hand side of (5.2) and we have proved that $\chi_{Q}(\bar{x})$ is more peaked than $\exp \left\{-\pi(\operatorname{det} \Omega)^{1 / N} \bar{x}^{T} \Omega^{-1} \bar{x}\right\}$ on $\boldsymbol{R}^{N}$. By the same method used to prove Theorem 1 we obtain

THeORem 7. Assume that the Conjecture 6 holds and let $A$ be a real $N \times K$ matrix, $\operatorname{rank}(A)=K$. Then

$$
(\operatorname{det} \Omega)^{-K / 2 N}\left|\operatorname{det} A^{T} \Omega^{-1} A\right|^{-1 / 2} \leqq \int_{R^{K}} \chi_{Q}(A \bar{x}) d \mu_{K}(\bar{x}) .
$$

If the set $Q$ in Theorem 7 is such that $\Omega$ is a constant multiple of the identity matrix then the left hand side of (5.3) is simply $\left|\operatorname{det} A^{T} A\right|^{-1 / 2}$. Just as in our proof of the corollary to Theorem 1, we deduce that in this case $\mu_{K}\left(Q \cap P_{K}\right) \geqq 1$, where $P_{K}$ is a $K$-dimensional subspace of $\boldsymbol{R}^{N}$. There is also an application of Theorem 7 to linear forms. If $L_{j}(\bar{x}), j=1,2, \cdots, N$, are $N$ linear forms in $K$ variables we could determine precise conditions under which

$$
\left(\sum_{j=1}^{N}\left|L_{j}(\bar{v})\right|^{p}\right)^{1 / p} \leqq \varepsilon
$$

at a nonzero lattice point $\bar{v}$ for any $p \geqq 1$ and $\varepsilon>0$. At present, however, these results remain hypothetical since they depend on the open problem stated in Conjecture 6.

\section{REFERENCES}

1. Z.W. Birnbaum, On random variables with comparable peakedness, Ann. Math. Stat., 19 (1948), 76-81.

2. C. Borell, Convex set functions in d-space, Period. Math. Hungar., 6 (1975), 111-136.

3. L. Breiman, Probability, Addison-Wesley, Reading, Mass., 1968.

4. J. W.S. Cassels, An Introduction to the Geometry of Numbers, Grundl. Math. Wiss. 99. Springer-Verlag, 1971, Berlin.

5. J. G. van der Corput, Verallgemeinerung einer Mordellschen Beweiismethode in der Geometrie der Zahlen, Acta Arith., 2 (1936), 145-146.

6. D. Hensley, Slicing the cube in $\boldsymbol{R}^{n}$ and probability, Proc. Amer. Math. Soc., (to appear).

7. M. Kanter, Unimodality and Dominance for symmetric random vectors, Trans. Amer. Math. Soc., 229 (1977), 65-85.

8. H. Minkowski, Geometrie der Zahlen, 1910, Leipzig.

9. A. Prekopa, On logarithmically concave measures and functions, Acta. Math. Acad. Hungar, (1973), 335-343.

10. S. Sherman, A theorem on convex sets with applications, Ann. Math. Stat., 26 (1955), 763-766. 
A GEOMETRIC INEQUALITY WITH APPLICATIONS TO LINEAR FORMS 553

Received November 21, 1978 and in revised form February 19, 1979. This research was supported by the National Science Foundation, grant number MCS77-01830.

The University of TeXas

AUSTIN, TX 78712 



\title{
PACIFIC JOURNAL OF MATHEMATICS
}

\section{EDITORS}

DONALD BABBITT (Managing Editor)

University of California

Los Angeles, CA 90024

\section{HUGO RossI}

University of Utah

Salt Lake City, UT 84112

C. C. MOORE and ANDREW OGG

University of California

Berkeley, CA 94720

\section{J. DUGUNDJI}

Department of Mathematics University of Southern California Los Angeles, CA 90007

R. FINN and J. Milgram

Stanford University Stanford, CA 94305

\section{ASSOCIATE EDITORS}
E. F. BECKENBACH
B. H. NeUmanN
F. WOLF
K. YosHIDA

\section{SUPPORTING INSTITUTIONS}

\author{
UNIVERSITY OF BRITISH COLUMBIA \\ CALIFORNIA INSTITUTE OF TECHNOLOGY \\ UNIVERSITY OF CALIFORNIA \\ MONTANA STATE UNIVERSITY \\ UNIVERSITY OF NEVADA, RENO \\ NEW MEXICO STATE UNIVERSITY \\ OREGON STATE UNIVERSITY \\ UNIVERSITY OF OREGON
}

\author{
UNIVERSITY OF SOUTHERN CALIFORNIA \\ STANFORD UNIVERSITY \\ UNIVERSITY OF HAWAII \\ UNIVERSITY OF TOKYO \\ UNIVERSITY OF UTAH \\ WASHINGTON STATE UNIVERSITY \\ UNIVERSITY OF WASHINGTON
}

The Supporting Institutions listed above contribute to the cost of publication of this Journal, but they are not owners or publishers and have no responsibility for its content or policies.

Mathematical papers intended for publication in the Pacific Journal of Mathematics should be in typed form or offset-reproduced, (not dittoed), double spaced with large margins. Please do not use built up fractions in the text of the manuscript. However, you may use them in the displayed equations. Underline Greek letters in red, German in green, and script in blue. The first paragraph or two must be capable of being used separately as a synopsis of the entire paper. Please propose a heading for the odd numbered pages of less than 35 characters. Manuscripts, in triplicate, may be sent to any one of the editors. Please classify according to the scheme of Math. Reviews, Index to Vol. 39. Supply name and address of author to whom proofs should be sent. All other communications should be addressed to the managing editor, or Elaine Barth, University of California, Los Angeles, California, 90024.

50 reprints to each author are provided free for each article, only if page charges have been substantially paid. Additional copies may be obtained at cost in multiples of 50 .

The Pacific Journal of Mathematics is issued monthly as of January 1966. Regular subscription rate: $\$ 84.00$ a year (6 Vols., 12 issues). Special rate: $\$ 42.00$ a year to individual members of supporting institutions.

Subscriptions, orders for numbers issued in the last three calendar years, and changes of address should be sent to Pacific Journal of Mathematics, P.O. Box 969, Carmel Valley, CA 93924, U.S.A. Older back numbers obtainable from Kraus Periodicals Co., Route 100, Millwood, NY 10546.

PUBLISHED BY PACIFIC JOURNAL OF MATHEMATICS, A NON-PROFIT CORPORATION

Printed at Kokusai Bunken Insatsusha (International Academic Printing Co., Ltd.). 8-8, 3-chome, Takadanobaba, Shinjuku-ku, Tokyo 160, Japan.

Copyright (C) 1979 by Pacific Journal of Mathematics Manufactured and first issued in Japan 


\section{Pacific Journal of Mathematics}

\section{Vol. 83, No. $2 \quad$ April, 1979}

Patrick Robert Ahern, On a theorem of Hayman concerning the derivative of a

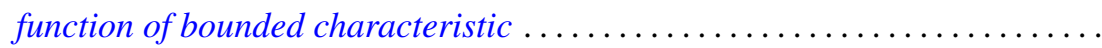

Walter Allegretto, Finiteness of lower spectra of a class of higher order elliptic

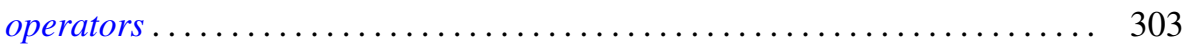

Leonard Asimow, Superharmonic interpolation in subspaces of $C_{c}(X) \ldots \ldots 11$

Steven F. Bellenot, An anti-open mapping theorem for Fréchet spaces . . . . . . . 325

B. J. Day, Locale geometry. . . . . . . . . . . . . . . . . . . . . . . . . . 333

John Erik Fornaess and Steven Krantz, Continuously varying peaking

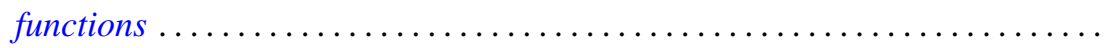

Joseph Leonide Gerver, Long walks in the plane with few collinear points ......

Joseph Leonide Gerver and Lawrence Thom Ramsey, On certain sequences of

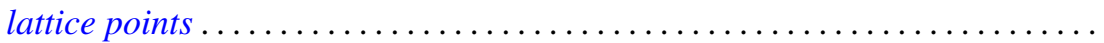

John R. Graef, Yuichi Kitamura, Takaŝi Kusano, Hiroshi Onose and Paul Winton

Spikes, On the nonoscillation of perturbed functional-differential

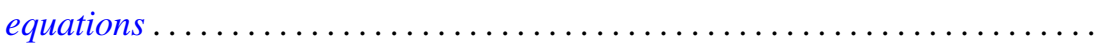

James A. Huckaba and James M. Keller, Annihilation of ideals in commutative

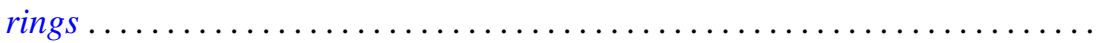

Anzelm Iwanik, Norm attaining operators on Lebesgue spaces . . . . . . . . . . .

Surjit Singh Khurana, Pointwise compactness and measurability . . . .......... 387

Charles Philip Lanski, Commutation with skew elements in rings with

involution.

Hugh Bardeen Maynard, A Radon-Nikodým theorem for finitely additive bounded

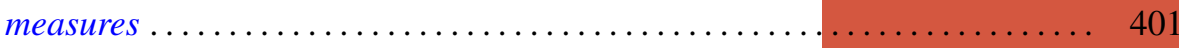

Kevin Mor McCrimmon, Peirce ideals in Jordan triple systems ..

Sam Bernard Nadler, Jr., Joseph E. Quinn and N. Stavrakas, Hyperspaces of compact convex sets.

Ken Nakamula, An explicit formula for the fundamental units of a real pure

sextic number field and its Galois closure ............

Vassili Nestoridis, Inner functions invariant connected components . .

Vladimir I. Oliker, On compact submanifolds with nondegenerate parallel

normal vector fields.

Lex Gerard Oversteegen, Fans and embeddings in the plane.

Shlomo Reisner, On Banach spaces having the property G.L

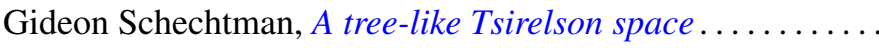

Helga Schirmer, Fix-finite homotopies . . . . . . . . . . . .

Jeffrey D. Vaaler, A geometric inequality with applications to linear forms . .

William Jennings Wickless, $T$ as an $\mathscr{G}$ submodule of $G$.....

Kenneth S. Williams, The class number of $Q(\sqrt{-p})$ modulo 4 , for $p \equiv 3$ (

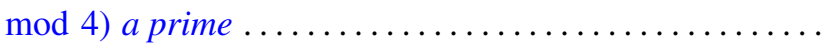

